NCL95-TP1

\title{
STOCHASTIC DYNAMICS OF LARGE-SCALE INFLATION IN DE SITTER SPACE
}

\author{
O. E. Buryak \\ Keldysh Institute of Applied Mathematics \\ Russian Academy of Sciences, Miusskaya Sq. 4 \\ Moscow 125047, Russia \\ and \\ Dept. of Physics, University of Newcastle \\ Newcastle upon Tyne NE1 $7 R U$, UK甘
}

\begin{abstract}
In this paper we derive exact quantum Langevin equations for stochastic dynamics of large-scale inflation in de Sitter space. These quantum Langevin equations are the equivalent of the Wigner equation and are described by a system of stochastic differential equations.

We present a formula for the calculation of the expectation value of a quantum operator whose Weyl symbol is a function of the large-scale inflation scalar field and its time derivative. The quantum expectation value is calculated as a mathematical expectation value over a stochastic process in an extended phase space, where the additional coordinate plays the role of a stochastic phase.

The unique solution is obtained for the Cauchy problem for the Wigner equation for large-scale inflation. The stationary solution for the Wigner equation is found for an arbitrary potential.

It is shown that the large-scale inflation scalar field in de Sitter space behaves as a quantum one-dimensional dissipative system, which supports the earlier results of Graziani and of Nakao, Nambu and Sasaki. But the analogy with a one-dimensional model of the quantum linearly damped anharmonic oscillator is not complete: the difference arises from the new time dependent commutation relation for the large-scale field and its time derivative.

It is found that, for the large-scale inflation scalar field the large time asymptotics is equal to the 'classical limit'. For the large time limit the quantum Langevin equations are just the classical stochastic Langevin equations (only the stationary state is defined by the quantum field theory).
\end{abstract}




\section{INTRODUCTION}

While the quasi-classical picture of the inflationary universe scenario, based on a FokkerPlanck evolution equation for the probability distribution of the inflation field, is almost complete (see the basic papers [1] 8] ), the essentially quantum-mechanical features of inflation are now a subject of investigation.

We would like to note recent investigations into this problem carried out by Graziani 9 [12, Nakao, Nambu and Sasaki [13, and Hu, Paz and Zhang [14. (The aim is not to present the full list of papers on this subject but to mention those which are closest to our considerations.)

In 13 the dynamics of an inflationary scalar field in a de Sitter background is investigated on the basis of the extended version of the stochastic approach proposed by Starobinsky [5]. In this approach, the scalar field operator is split into the long wavelength mode and the short wavelength mode. This split allows the reduction of the operator equation for the scalar field to Langevin equations of order $\sqrt{\hbar}$.

In the series of papers [9-12] of Graziani, dealing with quantum probability distributions and the dynamics of the early universe, the approach is based on the Wigner function and its evolution equation, which is the Wigner equation. The author concentrats attention on the large-scale inflation, where the spatial variable of the inflation scalar field is removed by averaging over a causal horizon volume. It is established that the quantum description of large-scale inflation in de Sitter space is equal to the quantum mechanics of a one-dimensional dissipative system. In [10] it is shown how quantum Langevin equations can be derived to any order of $\sqrt{\hbar}$ when they correspond to the Wigner equation expanded in powers of $\hbar$ and truncated at some power of $\hbar$. However the Wigner equation for large-scale inflation presented in 99 12 is not accurate and we will return to this point in our paper.

In paper [14] dealing with quantum Brownian motion $\mathrm{Hu}$, Paz and Zhang have derived an exact master equation for a quantum open system, which is an extension of earlier results obtained by Dekker [15], and Caldeira and Leggett [16]. As was established by Vilenkin [1], Linde [3] and Starobinsky [5], the basic stochastic equation for the large-scale quantum evolution of inflation is similar to Brownian motion, that is the quantum Langevin equations of order $\sqrt{\hbar}$. So the investigation of quantum Brownian motion is closely connected to further investigations of statistical and quantum effects at the early universe.

In this paper we restrict our consideration of the inflation scalar field to investigation of the dynamics of the large-scale or coarse-grained ( $\geq$ causal horizon) scalar field in a de Sitter background. Following in the main line Graziani [9 12] we describe the evolution of the large-scale inflation by the Wigner equation. Then the aim of this work is to derive

exact quantum Langevin equations (to all orders of $\sqrt{\hbar}$ ), which describe the stochastic dynamics of the large-scale inflation.

Thus, our approach is opposite in some sense to the approach of $\mathrm{Hu}$, Paz and Zhang [14. These authors move toward the possible observation of macroscopic effects from the search for an adequate description for statistical and quantum effects, while our way is to start from the macro-level to obtain an equivalent stochastic description. 


\section{BASIC FORMULATIONS}

The Lagrangian density of the inflation scalar field $\Phi(x, t)$ in a de Sitter background is

$$
\mathcal{L}=-\sqrt{-g}\left[\frac{1}{2} g^{\mu \nu} \partial_{\mu} \Phi \partial_{\nu} \Phi+\mathcal{V}(\Phi)\right],
$$

where the background metric is assumed to have the form

$$
d s^{2}=-d t^{2}+a^{2}(t) d x^{2},
$$

$g$ denotes a metric, $t$ is a time, $x$ is a three-dimensional spatial coordinate, $\mathcal{V}(\Phi)$ is a potential, and $a(t)$ is a scale factor (some positive function).

Then the equation for the classical inflation scalar field $\Phi(x, t)$ is

$$
\left[\frac{\partial^{2}}{\partial t^{2}}+3 \frac{\dot{a}(t)}{a(t)} \frac{\partial}{\partial t}-\left(\frac{1}{a(t)}\right)^{2} \Delta_{x}\right] \Phi(x, t)+\mathcal{V}^{\prime}[\Phi(x, t)]=0
$$

where $\Delta_{x}$ is the spatial Laplace operator, the dot over a symbol means a time derivative $\dot{y}(t)=d y / d t$, and $\mathcal{V}^{\prime}[\Phi]=d \mathcal{V}[\Phi] / d \Phi$.

The expansion of the universe is assumed,

$$
\frac{\dot{a}(t)}{a(t)}=H(t)
$$

where $H(t)$ is some non-negative integrable function of time. In other words, the scale factor is

$$
a(t)=a(0) \exp \left\{\int_{0}^{t} H(\tau) d \tau\right\}
$$

where $a(0)$ is the value of the scale factor at $t=0$, the beginning of inflation. In this model the beginning of inflation is taken to coincide with the origin of the universe.

Strictly speaking, in our consideration we deal with an expanding Friedmann-RobertsonWalker (FRW) universe, 'approximatly' close to a de Sitter universe in the sense: $H(t) \approx$ Const.

From (1) one obtains the canonical momentum conjugate to the field $\Phi(x, t)$ :

$$
\Pi(x, t)=\frac{\partial \mathcal{L}}{\partial(\partial \Phi / \partial t)}=a(t)^{3} \frac{\partial \Phi(x, t)}{\partial t}
$$

and the Hamiltonian density :

$$
\begin{aligned}
\mathrm{H} & =\Pi \frac{\partial \Phi}{\partial t}-\mathcal{L} \\
& =\frac{1}{2} a(t)^{-3} \Pi^{2}+\frac{1}{2} a(t)\left[\nabla_{x} \Phi\right]^{2}+a(t)^{3} \mathcal{V}(\Phi)
\end{aligned}
$$

When interested in large-scale ( $\geq$ causal horizon) physics, a coarse-graining procedure is utilised and it leads to a coarse-grained or averaged scalar field $\Phi_{X}(t)$ 


$$
\Phi_{X}(t)=\frac{1}{V} \int_{\Omega_{X}} \Phi(x, t) d x
$$

where the index $X$ is a label referring to the center of a region $\Omega$, over which $\Phi(x, t)$ is averaged, and $V$ is its volume. The volume of spatial averaging is taken not smaller than a causal horizon volume:

$$
V \geq \frac{4}{3} \pi \ell^{3}(t)
$$

with the causal horizon (or the 'coordinate horizon' in terms of Ellis [17] ) given by

$$
\ell(t)=\int_{0}^{t} a(\tau)^{-1} d \tau
$$

For de Sitter space

$$
a(t)=\exp \{H t\}, \quad H=\text { Const }
$$

then $\ell(t)=H^{-1}\left(1-e^{-H t}\right)$ and the volume of averaging is chosen to be $V=(4 / 3) \pi H^{-3}$.

For 'approximatly' de Sitter space we will think about a volume of averaging as constant for all time. This always can be assumed if $\ell(t) \leq$ Const. The techniques we use allow consider time dependent volume $V(t)$ along the same lines but we shall not consider this case for the sake of brevity. (See remarks in Section $\nabla \Pi 1$.)

Each large-scale region $\Omega$ (labeled by $X$ ) can be considered as a separate quantummechanical system because each lies outside of its neighbours' light cone: there is no exchange of information between large-scale regions. The profit of the coarse-graining procedure is that it reduces the quantum field problem to a quantum mechanical problem. At the same time there are still some peculiarities, following from the field theory, which do not make the analogy with quantum mechanics complete. We will point out these peculiarities in this section.

After performing the coarse-graining (8) the spatial varying term in the Euler- Lagrange eqation (3) can be neglected due to a smaller factor $a^{-2}(t) V^{-2 / 3}$ (see [18]) and the equation for the large-scale inflation field $\Phi_{X}(t)$ becomes

$$
\ddot{\Phi}_{X}(t)+3 H(t) \dot{\Phi}_{X}(t)+U^{\prime}\left[\Phi_{X}(t)\right]=0,
$$

where $U(\Phi)$ is the coarse-grained potential.

The averaging of the momentum (6) gives

$$
\Pi_{X}(t)=a(t)^{3} \dot{\Phi}_{X}(t)
$$

The Lagrangian (density) for the coarse-grained field $\Phi_{X}(t)$ is

$$
\mathcal{L}\left[\Phi_{X}\right]=a(t)^{3}\left[\frac{1}{2} \dot{\Phi}_{X}^{2}-U\left(\Phi_{X}\right)\right] .
$$

This gives the Euler-Lagrange equation (11) and keeps the averaged momentum (12) canonically conjugate to $\Phi_{X}(t)$ :

$$
\Pi_{X}(t)=\frac{\partial \mathcal{L}\left[\Phi_{X}\right]}{\partial \dot{\Phi}_{X}}
$$


Now the Hamiltonian (density) for the coarse-grained field $\Phi_{X}(t)$ is

$$
\mathcal{H}\left(\Phi_{X}, \Pi_{X} ; t\right)=\frac{1}{2} a(t)^{-3} \Pi_{X}^{2}+a(t)^{3} U\left(\Phi_{X}\right),
$$

it can be considered as the classical Hamiltonian for the large-scale inflation field, in the sense that the equations of motion produced by this Hamiltonian

$$
\frac{\partial \Phi_{X}}{\partial t}=\frac{\partial \mathcal{H}}{\partial \Pi_{X}}, \frac{\partial \Pi_{X}}{\partial t}=-\frac{\partial \mathcal{H}}{\partial \Phi_{X}}
$$

are equivalent to the field equation (111).

To make the step from the classical equation (11) to the quantum equation it is necessary to quantize (111) taking into account (12) and (15). This leads at the end to the quantum mechanics of a one-dimensional dissipative system in the description on $\Phi_{X}, \dot{\Phi}_{X}$ variables.

One can apply the canonical procedure, which is based on quantal noise operators and conserves the fundamental commutator for canonical position and momentum operators in the course of time, or the influence-functional method of Feynman and Vernon [19]. Both ways give the master equation for the 'reduced' density operator $\hat{\rho}$ (see [15, 16] for techniques), describing the time-evolution for the large-scale inflation,

$$
\frac{\partial \hat{\rho}}{\partial t}=\frac{V}{i \hbar}[\hat{\mathcal{H}}, \hat{\rho}]-\frac{V}{\hbar} a(t)^{6} D(t)\left[\hat{\Phi}_{X},\left[\hat{\Phi}_{X}, \hat{\rho}\right]\right] .
$$

Here $\hat{\mathcal{H}}$ is an operator form for the classical Hamiltonian (15), [, ] stands for a commutator, and the diffusion coefficient $D(t)$ is, in general, some non-negative function. In particular, the diffusion coefficient can be assumed to be $D(t)=3 H(t)$. Const, where Const is determined by physical parameters of the system at equilibrium.

Some one who would follow, formally, the quantisation procedure in quantum mechanics would notice immediately two points by which the master eqation (17) differs from the formally obtained one: firstly, the new constant $\hbar / V$ instead of Planck's constant $\hbar$, and secondly, the factor $a(t)^{6}$ in the diffusion term, which reflects the explicit time dependence of the Hamiltonian (15). It is time to discuss how these peculiarities come from the quantum field theory.

The origin of the scaling

$$
\hbar \rightarrow \hbar / V
$$

in equation (17), where $V$ is a volume of averaging (9), is that the fundamental commutator for canonical position and momentum operators in the relativistic quantum field theory

$$
[\hat{\Phi}(x, t), \hat{\Pi}(y, t)]=i \hbar \delta(x-y)
$$

is transformed by coarse-graining procedure (8) to

$$
\begin{gathered}
{\left[\hat{\Phi}_{X}(t), \hat{\Pi}_{X}(t)\right]=i \hbar / V,} \\
{\left[\hat{\Phi}_{X}(t), \hat{\Pi}_{Y}(t)\right]=0, \quad X \neq Y .}
\end{gathered}
$$


An other way to derive the master equation (17) would be to use the following momentum and Hamiltonian:

$$
\begin{aligned}
& \Pi_{\Omega}(t)=V \Pi_{X}(t), \\
& \mathcal{H}_{\Omega}=V \mathcal{H}\left(\Phi_{X}, \Pi_{X} ; t\right) .
\end{aligned}
$$

For momentum $\Pi_{\Omega}$ the fundamental commutator is

$$
\left[\hat{\Phi}_{X}(t), \hat{\Pi}_{\Omega}(t)\right]=i \hbar .
$$

The description in terms of (20)- (21) is natural for the large-scale region $\Omega$. Returning back to the spatially dependent field $\Phi(x, t)$, the Hamiltonian for $\Omega$ is found to be

$$
\mathcal{H}_{\Omega}=\int_{\Omega} \mathrm{H}(x, t) d x,
$$

or, in view of the Hamiltonian (density) (15),

$$
\mathcal{H}_{\Omega}=V \mathcal{H}\left(\Phi_{X}, \Pi_{X} ; t\right)
$$

(in (23) the spatially varying term of the original field $\left[\nabla_{x} \Phi\right]^{2}$ was neglected ).

Now let us discuss the diffusion term in the master equation (17). It should be mentioned that the general form of the diffusion term in the master equation is

$$
-\frac{V^{2}}{\hbar^{2}} C(t)[\hat{\Phi},[\hat{\Phi}, \hat{\rho}]],
$$

where $C(t)$ is some time-dependent coefficient. This coefficient is defined with respect to a suitably chosen vacuum state in the field theory. For de Sitter space it is the so-called Bunch- Davies vacuum [20]- [21]. In terms of (17) this means that it is determined by the physical parameters of the system at equilibrium.

If in accordance with the field theory (of inflation) we assume that the energy density of the equilibrium state is an invariant in de Sitter space then

$$
\left\langle\hat{\mathcal{H}}_{\Omega}(t)\right\rangle / a(t)^{3} V=\left\langle\hat{\mathcal{H}}\left(\hat{\Phi}_{X}, \hat{\Pi}_{X} ; t\right)\right\rangle / a(t)^{3}=\text { Const },
$$

where, to obtain the energy density, the Hamiltonian $\mathcal{H}_{\Omega}$ is divided by the proper volume $a(t)^{3} V$. The expectation values, \langle\rangle , of the operators are taken on the stationary solution of the master equation. For 'approximatly' de Sitter space, $\dot{H}(t)$ is neglected so the condition (24) can be assumed.

To fulfil (24) we have obtained the factor $a(t)^{6}$ in the diffusion term of (17). Taking into account that the constant in (24) is proportional to $(\hbar / V)$, in (17) we can show explicitly the dependence

$$
C(t)=\frac{\hbar}{V} a(t)^{6} D(t),
$$

which is valid for large-scale inflation. We will use master equation (17) as a starting point for investigation of the stochastic dynamics of the large-scale inflation.

From this point onwards in the paper the large-scale scalar field is refered to as $\Phi(t)$, omitting the index $X$. 


\section{THE WIGNER EQUATION FOR LARGE-SCALE INFLATION}

The Wigner function $W(q, p ; t)$ 22,23] is a function on the classical phase space and describes the distribution of position and momentum. The Wigner function is not a probability distribution since it can assume negative values; $W(q, p ; t)$ is a real function.

As a density matrix the Wigner function contains all of the information corresponding

to the quantum state. The expectation value for an arbitrary operator $\hat{A}(\hat{\Phi}, \hat{\Phi})$ can be calculated by the formula

$$
\langle\hat{A}\rangle=\int d q d p A(q, p) W(q, p ; t)
$$

where $A(q, p)$ is a Weyl symbol for the operator $\hat{A}(\hat{\Phi}, \hat{\dot{\Phi}})$.

Let us derive the time-evolution equation for the Wigner function for large-scale inflation, that is equivalent to the master equation (17).

For the Wigner operator $\hat{W}$ the equation has the same form as for the density operator $\hat{\rho}$ (17) (because coefficients $a(t)$ and $D(t)$ depend only on $t$ )

$$
\frac{\partial \hat{W}}{\partial t}=\frac{V}{i \hbar}[\hat{\mathcal{H}}, \hat{W}]-\frac{V}{\hbar} a(t)^{6} D(t)[\hat{q},[\hat{q}, \hat{W}]],
$$

where Weyl symbols for $\hat{W}$ and $\hat{\mathcal{H}}$ are

$$
\begin{gathered}
\hat{W} \leftrightarrow W(q, \Pi ; t), \\
\hat{\mathcal{H}} \leftrightarrow \mathcal{H}(q, \Pi)=\frac{1}{2} a(t)^{-3} \Pi^{2}+a(t)^{3} V(q) .
\end{gathered}
$$

Here we assume the following correspondence between phase space variables $(q, \Pi)$ or $(q, p)$ and variables of the large-scale inflation scalar field $(\Phi, \dot{\Phi})$ :

$$
\Phi=q, \quad \dot{\Phi}=p, \quad a(t)^{3} \dot{\Phi}=\Pi .
$$

To obtain an equation for the Wigner function $W(q, \Pi ; t)$ from its operator form (26) we will use the formula for the composition of operators in the Weyl formalism. Due to this formula [24] and the commutator relation (19) a Weyl symbol $A(q, \Pi)$ for the composition of two operators $\hat{A}=\hat{B} \hat{C}$ is defined via the correspondence

$$
\begin{aligned}
A(q, \Pi) & =B\left(\stackrel{I I}{q}+\frac{i \hbar}{2 V}^{I} \frac{\partial}{\partial \Pi}, \stackrel{I I}{\Pi}-\frac{i \hbar}{2 V}^{I} \frac{\partial}{\partial q}\right) C(q, \Pi) \\
& =C\left(\stackrel{I I}{q}-\frac{i \hbar^{I}}{2 V} \frac{\partial}{\partial \Pi}, \stackrel{I I}{\Pi}+\frac{i \hbar^{I}}{2 V} \frac{\partial}{\partial q}\right) B(q, \Pi)
\end{aligned}
$$

where $B(q, \Pi)$ and $C(q, \Pi)$ are Weyl symbols for the operators $\hat{B}$ and $\hat{C}$, and the numbers I,II over operators show the order in which the operators act.

Using (27), (29) one can obtain the following correspondence between operators and their Weyl symbols:

$$
\begin{aligned}
{[\hat{\mathcal{H}}, \hat{W}] \leftrightarrow } & -\frac{i \hbar}{V} a(t)^{-3} \Pi \frac{\partial}{\partial q} W(q, \Pi ; t) \\
& +a(t)^{3}\left[U\left(q+\frac{i \hbar}{2 V} \frac{\partial}{\partial \Pi}\right)-U\left(q-\frac{i \hbar}{2 V} \frac{\partial}{\partial \Pi}\right)\right] W(q, \Pi ; t),
\end{aligned}
$$




$$
[\hat{q},[\hat{q}, \hat{W}]] \leftrightarrow-\frac{\hbar^{2}}{V^{2}} \frac{\partial^{2}}{\partial \Pi^{2}} W(q, \Pi ; t) .
$$

Combining relations (30)-(31) in accordance with equation (26) one has the time evolution equation for the Wigner function $W(q, \Pi ; t)$

$$
\begin{aligned}
\frac{\partial W(q, \Pi ; t)}{\partial t}= & -a(t)^{-3} \Pi \frac{\partial W}{\partial q}+\frac{\hbar}{V} a(t)^{6} D(t) \frac{\partial^{2} W}{\partial \Pi^{2}} \\
& +\frac{V}{i \hbar} a(t)^{3}\left[U\left(q+\frac{i \hbar}{2 V} \frac{\partial}{\partial \Pi}\right)-U\left(q-\frac{i \hbar}{2 V} \frac{\partial}{\partial \Pi}\right)\right] W(q, \Pi ; t)
\end{aligned}
$$

Equation (32) is called the Wigner equation. (Note that the commonly used way to derive the Wigner equation is to apply the coordinate representation for the Wigner function

$$
W(q, \Pi ; t)=\frac{1}{2 \pi} \int_{-\infty}^{\infty} e^{i \Pi x}\left\langle q-\frac{\hbar}{2 V} x|\hat{\rho}| q+\frac{\hbar}{2 V} x\right\rangle d x
$$

to the master equation (17). Actually it is a longer way because it deals with multiple integrations and integrations by parts while the Weyl formalism gives an answer in a plain algebraic way.)

Because we are interested in the distribution of $\Phi$ and $\dot{\Phi}$ let us make the transition from $W(q, \Pi ; t)$ to $W(q, p ; t)$ by relations (28) and equality

$$
W(q, p ; t)=a(t)^{3} W(q, \Pi ; t)
$$

which follows from (25). For the Wigner function $W(q, p ; t)$ one has the time evolution equation

$$
\begin{aligned}
\frac{\partial W(q, p ; t)}{\partial t}= & -p \frac{\partial W}{\partial q}+3 H(t) \frac{\partial}{\partial p}(p W)+\frac{\hbar}{V} D(t) \frac{\partial^{2} W}{\partial p^{2}} \\
& +\frac{V}{i \hbar} a(t)^{3}\left[U\left(q+\frac{i \hbar}{2 V} a(t)^{-3} \frac{\partial}{\partial p}\right)-U\left(q-\frac{i \hbar}{2 V} a(t)^{-3} \frac{\partial}{\partial p}\right)\right] W(q, p ; t),
\end{aligned}
$$

where $H(t)$ is defined by (4).

The Wigner equation (35) can be rewritten in the following equivalent form:

$$
\begin{aligned}
\frac{\partial W(q, p ; t)}{\partial t}= & -p \frac{\partial W}{\partial q}+3 H(t) \frac{\partial}{\partial p}(p W)+\frac{\hbar}{V} D(t) \frac{\partial^{2} W}{\partial p^{2}} \\
& +\frac{V}{i \hbar} a(t)^{3} \int_{-\infty}^{\infty} d u W(q, p-u ; t) \cdot \mathcal{I}(q, u ; t),
\end{aligned}
$$

where

$$
\mathcal{I}(q, u ; t)=\frac{1}{2 \pi} \int_{-\infty}^{\infty} d y e^{-i u y}\left[U\left(q+\frac{\hbar}{2 V} a(t)^{-3} y\right)-U\left(q-\frac{\hbar}{2 V} a(t)^{-3} y\right)\right] .
$$

Equation (35), or equation (36), is the complete Wigner equation (to all orders of $\hbar$ ) for the large-scale inflation in de Sitter space. It describes the time evolution for the distribution of $\Phi$ and $\dot{\Phi}$ in a sense of equality (25). The expansion of the Universe, which is described 
by the scale factor $a(t)$ and equality (四), gives the origin for a dissipation term, with the disspation coefficient $3 H(t)$, in the Wigner equation. In this sense large-scale inflation can be considered as a quantum one-dimensional dissipative system, which supports Graziani's statement [9]. At the same time the expansion of the Universe influences the potential terms of equation (35) (or (36)), which was missed in [9 [12].

In [15, 14 the Wigner equation for a quantum disspative system was derived for a harmonic oscillator's potential $U(q)=\omega^{2} q^{2} / 2$. For an arbitrary potential $U(q)$ a truncated Wigner equation, or a Kramers-Moyal equation, is commonly used in the literature instead of the complete Wigner equation (see for example [16]). In our case (35) it would be

$$
\begin{aligned}
\frac{\partial W(q, p ; t)}{\partial t}= & -p \frac{\partial W}{\partial q}+3 H(t) \frac{\partial}{\partial p}(p W)+\frac{\hbar}{V} D(t) \frac{\partial^{2} W}{\partial p^{2}} \\
& +\frac{\partial U(q)}{\partial q} \cdot \frac{\partial W}{\partial p}+O\left(\hbar^{2}\right),
\end{aligned}
$$

where $O\left(\hbar^{2}\right)$ is a value of order $\hbar^{2}$.

The potential term in equation (35) can be expanded in powers of $\hbar$ by Taylor's series, which leads to equation

$$
\begin{aligned}
\frac{\partial W(q, p ; t)}{\partial t}= & -p \frac{\partial W}{\partial q}+3 H(t) \frac{\partial}{\partial p}(p W)+\frac{\hbar}{V} D(t) \frac{\partial^{2} W}{\partial p^{2}} \\
& +\sum_{k=1,3,5, \ldots}^{\infty} \frac{1}{k !}\left(\frac{i \hbar}{2 V} a(t)^{-3}\right)^{k-1} \frac{\partial^{k} U(q)}{\partial q^{k}} \frac{\partial^{k} W}{\partial p^{k}} .
\end{aligned}
$$

In the series of papers [9 12] the expansion in powers of $\hbar$ of the Wigner equation was used to show how to improve the accuracy of (38).

The potential term in Wigner equation (35) (or in the equivalent representations (36)(37) and (39) ) on a macro-level contains information about quantum noise on the micro-level, when

$$
\frac{\partial^{k} U(q)}{\partial q^{k}} \not \equiv 0 \quad \text { for } k \geq 3,
$$

This quantum noise is non-Gaussian noise. Condition (40) is always fulfilled when a potential deviates from a harmonic oscillator's potential. The diffusion term, containing $\partial^{2} W / \partial p^{2}$, in the Wigner equation also represents quantum noise (Gaussian noise) on a macro-level. Some authors consider a possibility that the Wigner equation may contain second order derivatives $\partial^{2} W / \partial q^{2}, \partial^{2} W / \partial q \partial p$, which are also of quantum origin [15, 14]. (These terms would correspond to additional terms $[\hat{\Pi},[\hat{\Pi}, \hat{\rho}]],[\hat{\Phi},[\hat{\Pi}, \hat{\rho}]],[\hat{\Pi},[\hat{\Phi}, \hat{\rho}]]$ in the master equation (17).) These terms have no physical sense because they lead to wrong Langevin equations: $d q_{t} / d t \neq p_{t}$ or $d \Phi / d t \neq \dot{\Phi}$.

In this paper we will work with the complete Wigner equation (36)-(37) for large-scale inflation to derive equations for the corresponding stochastic dynamics. Let us supply the evolution equation (36) with the initial condition

$$
W(q, p ; 0)=W_{0}(q, p),
$$


where the initial Wigner function is chosen to satisfy the following properties:

a) $W_{0}(q, p)$ is a real function,

b) $\quad \int W_{0}(q, p) d q d p=1$,

c) $\int\left[W_{0}(q, p)\right]^{2} d q d p \leq \frac{V}{2 \pi \hbar} a(0)^{3}$,

which follows from general properties for the Wigner function (see [23]).

\section{QUANTUM LANGEVIN EQUATIONS}

In this section we deduce quantum Langevin equations which are equal to the complete Wigner equation (36)-(37), but we start with some assumption about the potential $U(q)$.

$$
U(q)=\frac{\omega^{2}}{2} q^{2}+\int \exp \{-i q p\} \mu(d p)
$$

where $\mu(p)$ is a bounded measure such that

$$
\begin{aligned}
\int \mu(d p) & \leq \text { Const } \\
\int p^{2} \mu(d p) & \leq \text { Const. }
\end{aligned}
$$

The first term in the right hand side of equation (45) is just the harmonic oscillator's potential while the second term can be considered as a deviation from it. The potential is assumed to be real.

To include more model potentials used in the theory of inflation $\omega^{2}$ is allowed to be either positive or negative, or to be zero. A parabolic potential connected with 'chaotic inflation' is included in (45) with $\mu(d p) \equiv 0$. A double-well potential, representable in the form (45) is, for example

$$
U(q)=\omega^{2} q^{2} / 2+K \cos (k q) \cdot I_{[-(3 \pi / 2 k),(3 \pi / 2 k)]}(q),
$$

where $\omega, K, k$ are some parameters (real values) and $I_{A}(q)$ is the identificator of a set $A$ :

$$
I_{A}(q)= \begin{cases}1, & \text { if } q \in A \\ 0, & \text { if } q \notin A .\end{cases}
$$

Form (45) rules out model potentials which are polynomials higher than second order in $q$. Remember here that model potentials of polynomial type have appeared in the theory of inflation through expansion of $U(q)$ in powers of $q$ near $q=0$ (see, for example, [5]). Thus, almost all physical potentials for the inflation field $\Phi(t)$ can be represented as (45).

For potential (45) the Wigner equation becomes

$$
\begin{aligned}
\frac{\partial W(q, p ; t)}{\partial t}= & -p \frac{\partial W}{\partial q}+\omega^{2} q \frac{\partial W}{\partial p}+3 H(t) \frac{\partial}{\partial p}(p W)+\frac{\hbar}{V} D(t) \frac{\partial^{2} W}{\partial p^{2}} \\
& -\frac{i V}{\hbar} a(t)^{3} \int d \mu\left(p^{\prime}\right) \exp \left\{-i q p^{\prime}\right\}\left[W\left(q, p+\frac{\hbar}{2 V} a(t)^{-3} p^{\prime} ; t\right)\right. \\
& \left.-W\left(q, p-\frac{\hbar}{2 V} a(t)^{-3} p^{\prime} ; t\right)\right],
\end{aligned}
$$


$q, p, p^{\prime} \in \mathbf{R}^{1}, t \in[0, T]$, supplied by the initial condition

$$
W(q, p ; 0)=W_{0}(q, p)
$$

such that properties (42)-(44) are satisfied.

In order to solve this equation and to deduce stochastic equations, which describe a path in a phase space $(q, p) \equiv(\Phi, \dot{\Phi})$, we should reduce it to the form of a forward Kolmogorov equation 25] ( p.102). Such an approach was first proposed by Chebotarov and Maslov [26] and developed much further by Combe, Guerra et al. [27].

To reduce equation (48) to a forward Kolmogorov equation we need to transform the last term in the right hand side of it to a standard form. It can be done by introduction of a new function

$$
f(q, p, \theta ; t)=W(q, p ; t) \exp \left\{-\frac{2 V}{\hbar} A(t)+i \theta\right\}, \quad \theta \in \mathbf{R}^{1} / \bmod 2 \pi,
$$

where $\theta$ is a new variable, and function $A(t)$ will be defined later.

Inserting (50) into (48) one has

$$
\begin{aligned}
\frac{\partial f(q, p, \theta ; t)}{\partial t}= & -p \frac{\partial f}{\partial q}+\omega^{2} q \frac{\partial f}{\partial p}+3 H(t) \frac{\partial}{\partial p}(p f)+\frac{\hbar}{V} D(t) \frac{\partial^{2} f}{\partial p^{2}} \\
& +\frac{V}{\hbar} a(t)^{3} \int_{-\infty}^{\infty} d u \int \mu\left(d p^{\prime}\right)\left[f\left(q, p+\frac{\hbar}{2 V} a(t)^{-3} p^{\prime} u, \theta+\frac{\pi}{2} u-q p^{\prime} ; t\right)\right. \\
& -f(q, p, \theta ; t)] \cdot[\delta(u+1)+\delta(u-1)] \\
& +\frac{2 V}{\hbar} a(t)^{3} f(q, p, \theta ; t) \cdot \int \mu\left(d p^{\prime}\right)-\frac{2 V}{\hbar} \frac{d A(t)}{d t} f(q, p, \theta ; t),
\end{aligned}
$$

where $\delta(u)$ is a Dirac delta-function.

Let us put

$$
A(t)=\int_{0}^{t} a(\tau)^{3} d \tau \cdot \int \mu(d p) .
$$

Note that for scale factor $(10)$

$$
A(t)=\int \mu(d p) \cdot\left(e^{3 H t}-1\right) / 3 H .
$$

Let $m_{\hbar}(d p d u ; t)$ be the following measure:

$$
m_{\hbar}(d p d u ; t)=\frac{V}{\hbar} a(t)^{3} \mu(d p)[\delta(u+1)+\delta(u-1)] d u .
$$

The measure $m_{\hbar}(d p d u ; t)$ is a bounded measure on $\mathbf{R}^{1} \otimes(-1) \otimes(+1)$ multiplied by timedependent function $a(t)^{3}$. For an arbitrary function $\psi(p, u)$ an integral over this measure is

$$
\int \psi(p, u) m_{\hbar}(d p d u ; t)=\frac{V}{\hbar} a(t)^{3} \int[\psi(p,+1)+\psi(p,-1)] \mu(d p) .
$$


Now, with (52) and (54), equation (51) becomes

$$
\begin{aligned}
\frac{\partial f(q, p, \theta ; t)}{\partial t}= & -p \frac{\partial f}{\partial q}+\omega^{2} q \frac{\partial f}{\partial p}+3 H(t) \frac{\partial}{\partial p}(p f)+\frac{\hbar}{V} D(t) \frac{\partial^{2} f}{\partial p^{2}} \\
& +\int\left[f\left(q, p+\frac{\hbar}{2 V} a(t)^{-3} p^{\prime} u, \theta-\frac{\pi}{2} u-q p^{\prime} ; t\right)\right. \\
& -f(q, p, \theta ; t)] m_{\hbar}\left(d p^{\prime} d u ; t\right),
\end{aligned}
$$

which is the forward Kolmogorov equation.

If equation (56) is formally supplied by the initial condition

$$
f(q, p, \theta ; t)=\delta\left(q-q_{0}\right) \delta\left(p-p_{0}\right) \delta\left(\theta-\theta_{0}\right),
$$

then, according to the theory of stochastic differential equations (SDE), there exists a threedimensional stochastic process

$$
\xi_{t} \equiv\left(\Phi_{t}, \dot{\Phi}_{t}, \Theta_{t}\right)
$$

for which the function $f(q, p, \theta ; t)$ is a probability distribution. This means that for an arbitrary function $h(q, p, \theta)$, which is continuous and periodic with the period $2 \pi$ on variable $\theta$ and for fixed $\theta$ belongs to the class $\mathbf{C}_{b}^{1,2}\left(\mathbf{R}^{1} \otimes \mathbf{R}^{1}\right)$,

$$
\int h(q, p, \theta) f(q, p, \theta ; t) d q d p d \theta=\mathbf{E} h\left(\xi_{t}\right),
$$

where the symbol $\mathbf{E}$ denotes mathematical expectation and

$$
\xi_{t=0}=\left(q_{0}, p_{0}, \theta_{0}\right)
$$

(see equality (57)).

Using the generalized Ito formula for stochastic differentials (see [25],p.270, formula (13)) one deduces an SDE for the stochastic process $\xi_{t}(58)$

$$
\begin{aligned}
d \Phi_{t}= & \dot{\Phi}_{t} d t \\
d \dot{\Phi}_{t}= & -\left[3 H(t) \dot{\Phi}_{t}+\omega^{2} \Phi_{t}\right] d t+\left[\frac{2 \hbar}{V} D(t)\right]^{1 / 2} d w_{t} \\
& -\frac{\hbar}{2 V} a(t)^{-3} \int p u \nu_{\hbar}(d p d u ; d t), \\
d \Theta_{t}= & \int\left[\frac{\pi}{2} u+\Phi_{t} p\right] \nu_{\hbar}(d p d u ; d t) .
\end{aligned}
$$

Here $w_{t}$ is a one-dimensional Wiener process (or Brownian motion) and $\nu_{\hbar}(d p d u ; d t)$ is a Poisson measure on $\mathbf{R}^{\prime} \otimes(-1) \otimes(+1) \otimes[0, T]$ non-homogeneous with respect to translation on $[0, T]$ such that

$$
\mathbf{E}\left[\nu_{\hbar}(d p d u ; d t)\right]=m_{\hbar}(d p d u ; t) d t
$$

$\Theta_{t}$ is an additional stochastic variable, which can be interpreted as a stochastic phase (see equation (50)). It appears only due to deviation of the potential (45) from the harmonic oscillator's potential. 
In order for the stochastic process (61) -(63) to have a unique solution on a time interval $[0, T]$, right continuous with probability 1 , it is enough to have conditions (46), and to assume that functions $a(t), H(t), a(t)^{-3}, D(t)$ and their first derivatives are continuous functions.

Let us denote the stochastic process (61) $-($ 63) with initial condition (57) by

$$
\xi_{t}\left(q_{0}, p_{0}, \theta_{0}\right)=\left(\Phi_{t}\left(q_{0}, p_{0}, \theta_{0}\right), \dot{\Phi}_{t}\left(q_{0}, p_{0}, \theta_{0}\right), \Theta_{t}\left(q_{0}, p_{0}, \theta_{0}\right)\right) .
$$

If the initial condition for equation (56)

$$
f(q, p, \theta ; 0)=f_{0}(q, p, \theta)
$$

belongs to the class of generalized functions then instead of (59) one has

$$
\begin{aligned}
\int & h(q, p, \theta) f(q, p, \theta ; t) d q d p d \theta= \\
& =\int \mathbf{E} h\left(\Phi_{t}(q, p, \theta), \dot{\Phi}_{t}(q, p, \theta), \Theta_{t}(q, p, \theta)\right) f_{0}(q, p, \theta) d q d p d \theta .
\end{aligned}
$$

Now one can readily find the correspondence between an integral over the Wigner function $W(q, p ; t)$, governed by equation (48), and the stochastic process (61)-(63). Let us assume that

$$
f_{0}(q, p, \theta)=W_{0}(q, p)
$$

and let the function $h(q, p, \theta)$ in (67) be of the form

$$
h(q, p, \theta)=h(q, p) \exp \{-i \theta\} .
$$

Then from (67) and (50) one finds

$$
\begin{aligned}
& \int h(q, p) W(q, p ; t) d q d p= \\
& \quad=\exp \left\{\frac{2 V}{\hbar} A(t)\right\} \int \mathbf{E}\left\{h\left(\Phi_{t}(q, p, 0), \dot{\Phi}_{t}(q, p, 0)\right) \exp \left[-i \Theta_{t}(q, p, 0)\right]\right\} W_{0}(q, p) d q d p
\end{aligned}
$$

where $\int d \theta=2 \pi$ was used and the function $A(t)$ is defined by (52).

Formula (68) gives one the expectation value of a quantum operator $\hat{A}(\hat{\Phi}, \hat{\dot{\Phi}})$ with its Weyl symbol $h(q, p)$ (compare (68) with (25)).

Equations (61)- (63) are exact quantum Langevin equations for the large-scale inflation in de Sitter space associated with the master equation (17).

In [10] an attempt was made to derive the quantum Langevin equations for potentials with a polynomial growth higher than second order in $q$. As was mentioned, such potentials are excluded in our consideration, which is restricted by (45). In [10] the deduction of the quantum Langevin equations is based on a general expansion in powers of $\hbar$ of the Wigner equation, as in our representation (39). From [10] it follows that for polynomial potentials, of order higher than second, the quantum Langevin equations can be derived exactly only to order $\sqrt{\hbar / V}$ (corresponding to the truncated Wigner equation of order $\hbar / V$ ). Already the first correction of order $\hbar / V$ to the quantum Langevin equations cannot be calculated precisely (there is no explicit representation for the noise terms in the quantum Langevin 
equations). The origin of this problem is related to the term $\partial^{3} W / \partial p^{3}$ in the truncated Wigner equation of order $(\hbar / V)^{2}$.

Let us rewrite the quantum Langevin equations (61)-(63) in an integral form to show explicitly dependence on initial data $(q, p, 0)$ :

$$
\begin{aligned}
\Phi_{t}(q, p, 0)= & +\int_{0}^{t} \dot{\Phi}_{\tau}(q, p, 0) d \tau \\
\dot{\Phi}_{t}(q, p, 0)= & p-\int_{0}^{t}\left[3 H(\tau) \dot{\Phi}_{\tau}(q, p, 0)+\omega^{2} \Phi_{\tau}(q, p, 0)\right] d \tau+\left[\frac{2 \hbar}{V}\right]^{1 / 2} \int_{0}^{t} D(\tau)^{1 / 2} d w_{\tau} \\
& -\frac{\hbar}{2 V} \int_{0}^{t} a(\tau)^{-3} p^{\prime} u \nu_{\hbar}\left(d p^{\prime} d u ; d \tau\right), \\
\Theta_{t}(q, p, 0)= & \left\{\int_{0}^{t} \int\left[\frac{\pi}{2} u+\Phi_{\tau}(q, p, 0) p^{\prime}\right] \nu_{\hbar}\left(d p^{\prime} d u ; d \tau\right)\right\} / \bmod 2 \pi .
\end{aligned}
$$

Equations (69)-(71) describe a stochastic path $\Phi_{t}, \dot{\Phi}_{t}$ in phase space $(\Phi, \dot{\Phi})$ starting at a point $(q, p)$ when $t=0$. The stochastic phase $\Theta_{t}$ plays role in the final formula (68) for the expectation value of a quantum operator and can be interpreted as a contribution of the stochastic path $\Phi_{t}(q, p), \dot{\Phi}(q, p)$.

Equation (68) together with equations (61)-(63) can be used for numerical simulations to calculate the expectation value for quantum operator $\hat{A}(\hat{\Phi}, \hat{\dot{\Phi}})$, which is much simpler than solving the Wigner equation because SDE (61)-(63) are of first order.

\section{SOLUTION OF THE WIGNER EQUATION}

The aim of this section is to show how the Wigner function itself can be expressed by the expectation value with respect to a stochastic process in the extended phase space $(q, p, \theta)$. In [27] such an expression is found for the case

$$
\frac{\partial \hat{W}}{\partial t}=\frac{1}{i \hbar}[\hat{H}, \hat{W}], \quad H(q, p)=\frac{p^{2}}{2 m}+\frac{1}{2} m \omega^{2} q^{2}+U(q, p)
$$

while in the case under consideration (26), (15) the diffusion term and the expansion of the Universe play an essential role.

To find a solution for the Wigner equation (48) one should transform it to a backward Kolmogorov equation 25]( p.300). This can be done by introduction of the following function:

$$
f(q, p, \theta ; t)=W(q, p ; t) \exp \left\{-3 \int_{0}^{t} H(\tau) d \tau-\frac{2 V}{\hbar} A(t)+i \theta\right\}
$$

where $\theta \in \mathbf{R}^{1} / \bmod 2 \pi$, and $A(t)$ is the same function (52) as in Section $\mathbb{I V}$.

The time evolution of $f$ is governed now by the equation

$$
\begin{aligned}
\frac{\partial f(q, p, \theta ; t)}{\partial t}= & -p \frac{\partial f}{\partial q}+\left[\omega^{2} q+3 H(t) p\right] \frac{\partial f}{\partial p}+\frac{\hbar}{V} D(t) \frac{\partial^{2} f}{\partial p^{2}} \\
& +\int\left[f\left(q, p+\frac{\hbar}{2 V} a(t)^{-3} p^{\prime} u, \theta-\frac{\pi}{2} u-q p^{\prime} ; t\right)\right. \\
& -f(q, p, \theta ; t)] m_{\hbar}\left(d p^{\prime} d u ; t\right),
\end{aligned}
$$


which would be a backward Kolmogorov equation for the backward time $t^{\prime}$, by setting $t=T-t^{\prime}$, where $0 \leq t \leq T$. The measure $m_{\hbar}(d p d u ; t)$ is defined by (54).

If equation (74) is supplied by an initial condition

$$
f(q, p, \theta ; 0)=f_{0}(q, p, \theta)
$$

where $f_{0}$ is a continuous function, then the solution for (74) can be represented as

$$
f(q, p, \theta ; t)=\mathbf{E} f_{0}\left(Q_{T}(q, p, \theta ; T-t), P_{T}(q, p, \theta ; T-t), \Theta_{T}(q, p, \theta ; T-t)\right), \quad 0 \leq t \leq T
$$

where the stochastic process can be found by applying the generalized Ito formula for stochastic differentials 25] (p.270),

$$
\begin{aligned}
Q_{s}(q, p, \theta ; T-t)= & q-\int_{T-t}^{s} P_{\tau}(q, p, \theta ; T-t) d \tau \\
P_{s}(q, p, \theta ; T-t)= & p+\int_{T-t}^{s}\left[3 H(\tau) P_{\tau}(q, p, \theta ; T-t)+\omega^{2} Q_{\tau}(q, p, \theta ; T-t)\right] d \tau \\
& +\left[\frac{2 \hbar}{V}\right]^{1 / 2} \int_{T-t}^{s} D(\tau)^{1 / 2} d w_{\tau} \\
& +\frac{\hbar}{2 V} \int_{T-t}^{s} \int a(\tau)^{-3} p^{\prime} u \nu_{\hbar}\left(d p^{\prime} d u ; d \tau\right), \\
\Theta_{s}(q, p, \theta ; T-t)= & \left\{\theta-\int_{T-t}^{s} \int\left[\frac{\pi}{2} u+Q_{\tau}(q, p, \theta ; T-t) p^{\prime}\right] \nu_{\hbar}\left(d p^{\prime} d u ; d \tau\right)\right\} / \bmod 2 \pi
\end{aligned}
$$

where $0 \leq T-t \leq s \leq T$ and $\nu_{\hbar}\left(d p^{\prime} d u ; d \tau\right)$ is a Poisson measure on $\mathbf{R}^{1} \otimes(-1) \otimes(+1) \otimes[0, T]$ with the intensity defined by (64).

Returning to the Wigner function one has

$$
\begin{aligned}
W(q, p ; t)= & \exp \left\{3 \int_{0}^{t} H(\tau) d \tau+\frac{2 V}{\hbar} A(t)\right\} . \\
& \cdot \mathbf{E}\left\{W_{0}\left(Q_{T}(q, p, 0 ; T-t), P_{T}(q, p, 0 ; T-t)\right) \exp \left[i \Theta_{T}(q, p, 0 ; T-t)\right]\right\},
\end{aligned}
$$

where the stochastic process is defined by equations (77)-(79) with $\theta=0$.

Conditions for existence and uniqueness of the solution for SDE (77)-(79) are the same as for SDE (69)-(71).

Let us assume additionally that

$$
\int p^{k} \mu(d p) \leq \text { Const }, \quad k=3,4
$$

and that the initial Wigner function $W_{0}(q, p)$ is twice continuously differentiable in $p$ and once in $x$, and that its first and second order partials are bounded. Then the Wigner function (80) is twice continuously differentiable in $p$ and once in $x$, differentiable in $t$ and is the unique solution for the Cauchy problem (74)-(75).

Let us consider how properties (42)-(44) for the initial Wigner function are preserved in the course of time.

a) The reality of the Wigner function is preserved. To prove this proposition it is necessary to use the decomposition of the Wigner function into a difference of two positive 
functions (see [27 formula (4.4)). The group of transformation (80) preserves this decomposition.

b) The normalization for the Wigner function

$$
\int W(q, p ; t) d q d p=1, \quad t \geq 0,
$$

is fulfilled due to the equality

$$
\frac{d}{d t} \int W(q, p ; t) d q d p=0
$$

which follows directly from the Wigner equation.

c) The presence of the dissipation and diffusion terms in the Wigner equation destroys the inequality (44). It now becomes

$$
\int W^{2}(q, p ; t) d q d p \leq \int W_{0}^{2}(q, p) d q d p \cdot \exp \left\{3 \int_{0}^{t} H(\tau) d \tau\right\} \leq \frac{V}{2 \pi \hbar} a(t)^{3} .
$$

To derive (82) it is necessary to take a time derivative of the expression on the left hand side of (82) and to use the Wigner equation. After this one has

$$
\begin{aligned}
\int W^{2}(q, p ; t) d q d p= & {\left[\frac{a(t)}{a(0)}\right]^{3}\left\{\int W_{0}^{2}(q, p) d q d p\right.} \\
& \left.-\frac{2 \hbar}{V} \int_{0}^{t}\left[\frac{a(0)}{a(\tau)}\right]^{3} \cdot D(\tau) \cdot\left[\int\left(\frac{\partial W(q, p ; \tau)}{\partial p}\right)^{2} d q d p\right] d \tau\right\}
\end{aligned}
$$

and (82) follows from $(83)$.

If there is no diffusion, $D(t) \equiv 0$, for such a system, starting at $t=0$ from a pure state

$$
\int\left[W_{0}(q, p)\right]^{2} d q d p=\frac{V}{2 \pi \hbar} a(0)^{3}\left(<\frac{V}{2 \pi \hbar} a(0)^{3} \text { for a mixed state }\right)
$$

it is possible to follow the pure state in the course of time due to equality

$$
\int W^{2}(q, p ; t) d q d p=\frac{V}{2 \pi \hbar} a(t)^{3}\left(<\frac{V}{2 \pi \hbar} a(t)^{3} \text { for a mixed state }\right) .
$$

However, diffusion $(D(t) \not \equiv 0)$ smears the picture and one cannot disinguish pure and mixed states by inequality (82).

For the Wigner function $W(q, \Pi ; t)$ (see (34)) relations (83), (82) become

$$
\begin{aligned}
\int W^{2}(q, \Pi ; t) d q d \Pi= & \int W_{0}^{2}(q, \Pi) d q d \Pi \\
& -\frac{2 \hbar}{V} \int_{0}^{t} a^{6}(\tau) D(\tau)\left[\int\left(\frac{\partial W(q, \Pi ; \tau)}{\partial \Pi}\right)^{2} d q d \Pi\right] d \tau, \\
\int W^{2}(q, \Pi ; t) d q d \Pi \leq & \frac{V}{2 \pi \hbar} .
\end{aligned}
$$




\section{LARGE TIME ASYMPTOTICS}

If $H(t) \approx$ Const the scale factor $a(t)$ increases exponentially with time and for large time the Wigner equation becomes

$$
\frac{\partial W(q, p ; t)}{\partial t}=-p \frac{\partial W}{\partial q}+3 H(t) \frac{\partial}{\partial p}(p W)+\frac{\partial U(q)}{\partial q} \cdot \frac{\partial W}{\partial p}+\frac{\hbar}{V} D(t) \frac{\partial^{2} W}{\partial p^{2}}
$$

to order $a(t)^{-3}$, what follows from (39).

The expectation value at large time $T$ for an operator $\hat{A}$ with Weyl symbol $h(q, p)$ is

$$
\begin{aligned}
\langle\hat{A}\rangle_{T} & =\int h(q, p) W(q, p ; T) d q d p \\
& =\int \mathbf{E} h\left(\Phi_{T}(q, p, 0), \dot{\Phi}_{T}(q, p, 0)\right) W_{0}(q, p) d q d p
\end{aligned}
$$

where the stochastic process $\left(\Phi_{t}, \dot{\Phi}_{t}\right)$ is governed now by

$$
\begin{aligned}
& d \Phi_{t}=\dot{\Phi}_{t} d t \\
& d \dot{\Phi}_{t}=-\left[3 H(t) \dot{\Phi}_{t}+\dot{U}\left(\Phi_{t}\right)\right] d t+\left[\frac{2 \hbar}{V} D(t)\right]^{1 / 2} d w_{t}
\end{aligned}
$$

with the initial condition $\left(\Phi_{0}, \dot{\Phi}_{0}\right)=(q, p)$.

Note that the stochastic differentials (89) are equivalent to the quantum Langevin equations (61) - 63) for the scalar field $\Phi(t)$ in the large time limit (not for the beginning of the inflation if the potential $U(q)$ satisffies the unequality (40).

\section{STATIONARY STATES FOR LARGE-SCALE INFLATION}

Stationary or equilibrium states for large-scale inflation are described by the stationary Wigner function $W(q, p)=\lim _{t \rightarrow \infty} W(q, p ; t)$ governed by the Wigner equation

$$
0=-p \frac{\partial W}{\partial q}+3 H(t) \frac{\partial}{\partial p}(p W)+\frac{\partial U(q)}{\partial q} \cdot \frac{\partial W}{\partial p}+\frac{\hbar}{V} D(t) \frac{\partial^{2} W}{\partial p^{2}}
$$

for an arbitrary potential $U(q)$. This equation has time dependent coefficients coming from the time dependence of the scale factor.

For the stationary Wigner function $W(q, p)$ one can easily find the following expectation values for the operators:

$$
\begin{aligned}
\langle\hat{\dot{\Phi}}\rangle & =0, \\
\langle\hat{\dot{U}}(\hat{\Phi})\rangle & =0, \\
\left\langle\hat{\dot{\Phi}}^{2}\right\rangle & =\langle\hat{\Phi} \hat{\dot{U}}(\hat{\Phi})\rangle=\frac{\hbar D(t)}{V \cdot 3 H(t)} .
\end{aligned}
$$

From the last equality one can see that the diffusion coefficient $D(t)$ should be

$$
D(t)=3 H(t) \sigma
$$


(at least for large time), where the constant $\sigma$ is defined by the choice of stationary state:

$$
\left\langle\hat{\dot{\Phi}}^{2}\right\rangle=\frac{\hbar}{V} \sigma
$$

The stationary Wigner function is found to be

$$
W(q, p)=N \exp \left\{-\left(\frac{p^{2}}{2}+U(q)\right) V /(\hbar \sigma)\right\}
$$

where $\mathrm{N}$ is the normalization constant.

For a particular case, with the scale factor given by (10) and potential $U(q)=\omega^{2} q^{2} / 2$, the Bunch-Davies vacuum is given by

$$
\left\langle\hat{\Phi}^{2}\right\rangle=\frac{3 H^{4} \hbar}{8 \pi^{2} \omega^{2}}=\frac{\hbar H}{V 2 \pi \omega^{2}}
$$

which yields $\sigma=H / 2 \pi$ and $D=3 H^{2} / 2 \pi$.

For this case the stationary solution is

$$
W(q, p)=\frac{V \omega}{\hbar H} \exp \left\{-\left(p^{2}+\omega^{2} q^{2}\right) \frac{V \pi}{\hbar H}\right\}, \quad V=\frac{4 \pi}{3 H^{3}},
$$

where one must assume that

$$
H \geq \omega / \pi
$$

for the inequality (44) to be satisfied by the Wigner function (98).

\section{CONCLUSIONS}

1. The appearance of the dissipation term $3 H(t)(\partial / \partial p)(p W(q, p ; t))$ in the Wigner equation, after transition from phase space $(\Phi, \Pi)$ to $(\Phi, \dot{\Phi})$, supports the earlier result of Graziani [9] and Nakao, Nambu and Sasaki [13], that the large-scale inflation scalar field behaves as a quantum one-dimensional dissipative system. Nevertheless, this analogy is not complete: it is destroyed by a new commutation relation

$$
[\hat{\Phi}, \hat{\dot{\Phi}}]=\frac{i \hbar}{V} a(t)^{-3}
$$

where $a(t)$ is the scale factor in de Sitter metric and it reflects the expansion of the Universe $($ see (4) $)$.

Comparing the Wigner equation (48) for the large-scale inflation (or more general case (36)-(37)) with the Wigner equation for a quantum one-dimensional linearly damped unharmonic oscillator (see [14 16]), one can see that the expansion of the Universe amplifies the role of the potential term, which is a deviation from the harmonic oscillator potential. Explicitly this amplification appears in formula (68) for the expectation value of a quantum operator and in formula (80) for the Wigner function, where function $A(t)$ (52) has the factor $\int_{0}^{t} a(\tau)^{3} d \tau$ (without expasion this factor would be 1 ). At the same time, as $t \rightarrow \infty$, 
the commutation relation (101) leads to degeneration of the jump process, in the quantum Langevin equations, into a continuous process (see Section VI).

2. As a consequence of our investigation we have the following statement: for the largescale inflation scalar field the asymptotic $t \rightarrow \infty$ is equal to the classical limit.

In the limit $\hbar \rightarrow 0$ the Wigner equation (36)-(37) (or (48)) turns out to be

$$
\frac{\partial W(q, p ; t)}{\partial t}=-p \frac{\partial W}{\partial q}+3 H(t) \frac{\partial}{\partial p}(p W)+\frac{\partial U(q)}{\partial q} \cdot \frac{\partial W}{\partial p}
$$

and the corresponding Langevin equations are

$$
\begin{aligned}
d \Phi_{t} & =\dot{\Phi}_{t} d t \\
d \dot{\Phi}_{t} & =-\left[3 H(t) \dot{\Phi}_{t}+U^{\prime}\left(\Phi_{t}\right)\right] d t .
\end{aligned}
$$

Equations (102) are just the classical deterministic equations of motion, equivalent to the field equation (11).

However, (101) is not the 'classical limit' for the Wigner equation (36)- (37) (only the truncated Wigner equation of order $\hbar_{0}$. In the classical limit the diffusion term in the Wigner equation (36) - (37) is not proportional to $\hbar / V$ because, instead of (95) for a stationary state, one has

$$
\left\langle\dot{\Phi}^{2}\right\rangle=\sigma_{c l}
$$

The 'classical limit' of the Wigner equation for the large-scale inflation scalar field is

$$
\frac{\partial W(q, p ; t)}{\partial t}=-p \frac{\partial W}{\partial q}+3 H(t) \frac{\partial}{\partial p}(p W)+\frac{\partial U(q)}{\partial q} \cdot \frac{\partial W}{\partial p}+D_{c l}(t) \frac{\partial^{2} W}{\partial p^{2}}
$$

with $D_{c l}(t)=3 H(t) \sigma_{c l}$. The classical stochastic Langevin equations are

$$
\begin{aligned}
d \Phi_{t} & =\dot{\Phi}_{t} d t \\
d \dot{\Phi}_{t} & =-\left[3 H(t) \dot{\Phi}_{t}+U^{\prime}\left(\Phi_{t}\right)\right] d t+\left[2 D_{c l}(t)\right]^{1 / 2} d w_{t} .
\end{aligned}
$$

One can see that that in the 'classical limit' the Wigner equation and Langevin equations ((104) and (105)) are the same as those of the large time asymptotic ( equations (87) and (89)). For complete coincidence the coefficient of the second derivative in the Wigner equations (87) and (104) should be presented in the form $3 H(t)\left\langle\dot{\Phi}^{2}\right\rangle_{s t}$, where the expectation value $\left\langle\dot{\Phi}^{2}\right\rangle_{s t}$ is taken on the corresponding stationary state.

3. In this paper each large-scale region (for the coarse-graining procedure) is considered as an independent quantum mechanical system. If, nevertheless, it is necessary to take into account interaction with the environment, then for linear interaction the master equation for the 'reduced' density operator $\hat{\rho}$ is

$$
\begin{aligned}
\frac{\partial \hat{\rho}}{\partial t}= & \frac{V}{i \hbar}[\hat{\mathcal{H}}, \hat{\rho}]+\frac{\lambda(t) V}{4 i \hbar}[\{\hat{\Pi}, \hat{\Phi}\}, \hat{\rho}] \\
& +\frac{\lambda(t) V}{2 i \hbar}([\hat{\Phi}, \hat{\rho} \hat{\Pi}]-[\hat{\Pi}, \hat{\rho} \hat{\Phi}])-\frac{V}{\hbar} a(t)^{6} D(t)[\hat{\Phi},[\hat{\Phi}, \hat{\rho}]]
\end{aligned}
$$


where $\lambda(t)$ is the dissipation coefficient (originated by interaction with the environment), $\hat{\mathcal{H}}$ is an operator form for the Hamiltonian (15) of the system without dissipation, and $\{$, stands for an anti-commutator.

In phase space $(\Phi, \dot{\Phi})$ the Wigner equation corresponding to $(\mathbb{1 0 6})$ is

$$
\begin{aligned}
\frac{\partial W(q, p ; t)}{\partial t}= & -p \frac{\partial W}{\partial q}+[3 H(t)+\lambda(t)] \frac{\partial}{\partial p}(p W)+\frac{\hbar}{V} D(t) \times \\
& \times \frac{\partial^{2} W}{\partial p^{2}}+\frac{V}{i \hbar} a(t)^{3} \int_{-\infty}^{\infty} d u W(q, p-u ; t) \mathcal{I}(q, u ; t),
\end{aligned}
$$

where $\mathcal{I}(q, u ; t)$ is defined by (37).

Equation (107) is an equation of the same type as the Wigner equation (36). Thus, (107) can be treated as the Wigner equation considered in this paper.

4. Since in our consideration on phase space $(\Phi, \dot{\Phi})$ the scaling $\hbar a(t)^{-3} / V$ depends already on $t$, it is not a problem to take into account a time dependence of the coarse-graining volume $V(t)$. The scaling becomes $\hbar a(t)^{-3} / V(t)$, where $V(0)=$ Const $\neq 0$ is assumed.

Therefor the result can be extended on an expanding FRW space-time. The principal point in this case is the new time dependence of the canonical momentum conjugate to $\Phi_{X}(t)$ :

$$
\Pi_{\Omega}(t)=V(t) a(t)^{3} \dot{\Phi}_{X}(t)
$$

and of the Hamiltonian:

$$
\mathcal{H}_{\Omega}=\frac{1}{2} V(t)^{-1} a(t)^{-3} \Pi_{\Omega}^{2}+V(t) a(t)^{3} U\left(\Phi_{X}\right)
$$

(compare with (20) and (23)). Also the condition (24), taken for 'approximatly' de Sitter space, should be replaced by a condition corresponding to each concrete FRW space-time.

\section{ACKNOWLEDGMENTS}

The support of the Royal Society via a postdoctoral fellowship and the partial support of the Russian Academy of Sciences under grant No 94-02-06688 are acknowledged. The author is very grateful to Dr. Ian G. Moss for constructive advice and discussions, and to the mathematical physics group of the University of Newcastle upon Tyne for a very fruitful and supportive atmosphere. 


\section{REFERENCES}

$\dagger \quad$ Electronic mail: Olga.Buryak@newcastle.ac.uk (until June 1995); Buryak@applmat.msk.su.

[1] A. Vilenkin, Phys. Rev. D27, 1848 (1983)

[2] A. H. Guth and S.-Y. Pi, Phys. Rev. D32, 1899 (1985).

[3] A. D. Linde, Phys. Lett. B175, 395 (1986).

[4] A. S. Goncharov and A. D. Linde, Sov. J. Part. Nucl. 17, 369 (1986).

[5] A. A. Starobinsky, in Current Topics in Field Theory, Quantum Gravity, and Strings, proceedings of the VI conference, Meudon and Paris, edited by H. J. de Vega and N. Sànchez (Lecture Notes in Physics, Vol. 246) (Springer, New York, 1986), p. 107.

[6] J. M. Bardeen and G. J. Bublik, Class. Quantum Gravit. 4, 673 (1987).

[7] S.-J. Rey, Nucl. Phys. B284, 706 (1987).

[8] M. Aryal and A. Vilenkin, Phys. Lett. 199B, 351 (1987).

[9] F. R. Graziani, Phys. Rev. D38, 1122 (1988).

[10] F. R. Graziani, Phys. Rev. D38, 1131 (1988).

[11] F. R. Graziani, Phys. Rev. D38, 1802 (1988).

[12] F. R. Graziani, Phys. Rev. D38, 3630 (1989).

[13] K.-I. Nakao, Y. Nambu, and M. Sasaki, Prog. Theor. Phys. 80, 1041 (1988).

[14] B. L. Hu, J. P. Paz, and Y. Zhang, Phys. Rev. D48, 2843 (1992).

[15] H. Dekker, Phys. Rev. A16, 2116 (1977).

[16] A. O. Caldeira and A. J. Leggett, Physica 121A, 587 (1983).

[17] G. F. R. Ellis and T. Rothman, Am. J. Phys. Vol.61, No 10, 883 (1993).

[18] S. K. Ma, Modern Theory of Critical Phenomena (Benjamin/Cummings, New York, 1976).

[19] R. P. Feynman and F. L. Vernon, Annals of Physics 24, 118 (1963).

[20] T. S. Bunch and P. C. W. Davies, Proc. R. Soc. London A 360, 117 (1978).

[21] N. D. Birrell, Proc. R. Soc. London A 361, 1315 (1978).

[22] E. Wigner, Phys. Rev. 40, 749 (1932).

[23] M. Hillery, R. F. O'Connell, M. O. Scully and E. P. Wigner, Phys. Rep. 106, 121 (1984).

[24] V. P. Maslov, Matematicheskie Zametki (in Russian), Vol. 46, No 1, p. 37-52 (1989).

[25] I. I. Gihman, A. V. Skorohod, Stochastic Differential Equations (Springer-Verlag, Berlin, Heidelberg, New York, 1972).

[26] V. P. Maslov and A. M. Chebotarev, Lect. Notes in Physics B, Vol. 106 (SpringerVerlag, Berlin, 1979), p. 58.

[27] Ph. Comber, F. Guerra, R. Rodriguez, M. Sirigue, M. Sigue-Collin, Physica 124 A, 561 (1984). 